\title{
Gamma-Ray Effects of Dark Forces in Dark Matter Clumps
}

\author{
K. Belotsky, ${ }^{1,2}$ M. Khlopov, ${ }^{1,2,3}$ and A. Kirillov ${ }^{1,2}$ \\ ${ }^{1}$ National Research Nuclear University "MEPhI", Moscow 115409, Russia \\ ${ }^{2}$ Centre for Cosmoparticle Physics "Cosmion", Moscow 115409, Russia \\ ${ }^{3}$ APC Laboratory, 10 rue Alice Domon et Léonie Duquet, 75205 Paris Cedex 13, France
}

Correspondence should be addressed to A. Kirillov; kirillov-aa@yandex.ru

Received 28 October 2013; Revised 20 December 2013; Accepted 2 April 2014; Published 23 April 2014

Academic Editor: Jean-René Cudell

Copyright (C) 2014 K. Belotsky et al. This is an open access article distributed under the Creative Commons Attribution License, which permits unrestricted use, distribution, and reproduction in any medium, provided the original work is properly cited. The publication of this article was funded by SCOAP S $^{3}$

Existence of new gauge U(1) symmetry possessed by dark matter (DM) particles implies the existence of a new Coulomb-like interaction, which leads to Sommerfeld-Gamow-Sakharov enhancement of dark matter annihilation at low relative velocities. We discuss a possibility to put constraints on such dark forces of dark matter from the observational data on the gamma radiation in our Galaxy. Gamma-rays are supposed to originate from annihilation of DM particles in the small scale clumps, in which annihilation rate is supposed to be enhanced, besides higher density, due to smaller relative velocities $v$ of DM particles. For possible cross sections, mass of annihilating particles, masses of clumps, and the contribution of annihilating particles in the total DM density we constrain the strength of new dark long range forces from comparison of predicted gamma-ray signal with Fermi/LAT data on unidentified point-like gamma-ray sources (PGS) as well as on diffuse $\gamma$-radiation. Both data on diffuse radiation and data on PGS put lower constraints on annihilation cross section at any dark interaction constant, where diffuse radiation provides stronger constraint at smaller clump mass. Density of annihilating DM particles is conventionally supposed to be defined by the frozen annihilation processes in early Universe.

\section{Introduction}

From the first articles revealing the indirect effects of the cold dark matter (CDM) in the form of heavy neutral leptons [1$8]$ or supersymmetric particles $[7,9,10]$, indirect effects of dark matter annihilation had been the subject of intensive studies in the data on the cosmic rays (CR) and gamma radiation. In the CDM scenario DM particles could form the hierarchic structures over a wide range of scales and masses (from small scale clumps to large scale structures) [11-16]. The annihilation rate of DM particles within these clumpy structures, giving rise to cosmic ray signals $[4-8,17-25]$, should be enhanced due to higher density of DM particles in them, as compared with their averaged density in the Galaxy. The annihilation cross section can be also enhanced at small relative velocities of DM particles, which are especially small in the lightest clumps, which are likely to be the most abundant. Due to these factors the clumps, located in a neighborhood of Solar System, can be observed as discrete (basically point-like) gamma-ray sources $[19,21,26-33]$, while the overall effect of such clumps should increase the diffuse gamma background in the Galaxy.

In this paper we address the sensitivity of the given effect [31-33] to the existence of long range dark forces originated from new $\mathrm{U}(1)$ gauge charges, which annihilating dark matter particles possess. Coulomb-like interaction (we will refer to it as " $y$-interaction") of these $\mathrm{U}(1)$ charges leads to Sommerfeld-Gamow-Sakharov (SGS) enhancement of particle annihilation at low relative velocities. Since small scale clumps of dark matter have small gravitational potential, the relative velocities of particles, annihilating within these clumps, are much smaller than those of unclumped particles in the galactic halo, which enhances the sensitivity of both the data on the discrete gamma-ray sources and the data on the diffuse $\gamma$-radiation to the effects of the dark forces in dark matter annihilation.

One should note that effects of DM annihilation enhancement due to new interaction were first considered in $[34,35]$ and then in many successive papers, for example, [36-40]. In most of these papers SGS enhancement was mainly 
studied for the case of dominant form of DM that possesses new interaction with massive carrier, and the effect of this enhancement for point-like gamma-ray sources, treated in the present work, was not considered.

The existence of new U(1) gauge symmetry implies the existence of the corresponding massless dark photons. They can increase the effective number of relativistic species at the radiation dominated stage. Decoupling of such photons from the plasma in the early Universe takes place in the period of freezing out of the CDM particles, which makes this contribution compatible with the measurements of the effective number of the neutrino species (see, e.g., [41] for review and references).

Note that new interaction can lead to binding of DM particles into atomic-like states with their successive annihilation [41], or stable dark atoms in case of multicomponent (charge asymmetric) DM [41-43]. This effect, going beyond the scope of the present work, deserves separate consideration.

\section{Gamma-Ray Signal from $y$-Interacting DM Clumps}

In calculations of gamma-ray signals from the clump in this paper we follow our previous work [31-33]. For density profile inside the clumps we use profile BGZ obtained in $[19,21,26]$, which gives the minimal estimation of the $\gamma$-flux from the clump [31-33]. Fraction $\xi=0.002$ from total density of surrounding DM is taken for the clumps that survived until present time. We study $\gamma$-radiation effect for only minimal clump mass, formally assuming that all DM clumps are with this mass. According to theoretical estimates consistent with observations, the small mass clumps are predicted to be the most abundant $[19,21,44]$. However, the following merging and formation of the high-mass clumps (subhalos) lead to the transformation of the mass distribution and the subhalos effect can be also noticeable [45], but the picture does not change a lot.

To cover a wide class of models of DM particles, we parametrize their annihilation cross section as follows:

$$
\sigma_{\mathrm{ann}}=\frac{\sigma_{0}}{v} \times C(v, \alpha)
$$

with $v$ being relative velocity of annihilating DM particle and antiparticle. Parameter $\sigma_{0}$ is determined by cosmological density of the particles $\Omega$. The factor $C(v, \alpha)$ takes explicitly into account a possible Coulomb-like $y$-interaction of DM particles, which leads to a Sommerfeld-Gamow-Sakharov enhancement [46-48] and has the form

$$
C(\nu, \alpha)=\frac{2 \pi \alpha / v}{1-\exp (-2 \pi \alpha / v)}
$$

Here $\alpha$ is the fine structure constant of additional interaction.

One should note that $y$-interaction not only implies SGS enhancement of the annihilation cross section but also leads to the existence of new channels that involve $y$-photons in the final state. The corresponding suppression of the branching ratio for ordinary photon production is effectively taken into account in our calculations by a multiplicity of produced photons, $N_{\gamma}$ (see below).

The enhancement (2) of the annihilation cross section may lead to decrease of the frozen out density of DM particles. However in the period of their freezing out in the early Universe the particles were semirelativistic (with typical velocities $v \sim c / 5$ ) so that relic density cannot decrease significantly. Effect of annihilation of DM particles in massless bosons of $y$-interaction ( $y$-photons) not only needs in general special study in the framework of particular models of DM particles but it also cannot strongly increase the annihilation cross section (and correspondingly decrease the relic abundance) taking into account all the other possible annihilation channels.

In the modern Universe, when the particle velocities are nonrelativistic, factor (2) may significantly enhance the annihilation effects [49-52]. Such effects become noticeable even for a subdominant component with $\Omega \ll \Omega_{\mathrm{CDM}}$ as it takes place in case of heavy stable neutrinos with $y$-interaction [53]. Therefore we suppose that an active (annihilating) component of DM may be both dominant and subdominant; that is, $\Omega \leq \Omega_{\mathrm{CDM}}$ with $\Omega_{\mathrm{CDM}} \sim 0.2$ being the total relative density of cold dark matter in Universe. In estimation of cosmological density $\Omega$ we follow the standard approach $[54,55]$.

It is worth noting that the given scheme does not take into account possibility of binding pairs of considered particle-antiparticle due to $y$-interaction. If nonrelativistic DM particles are decoupled from the ambient plasma, their rapid cooling can strongly enhance the rate of such recombination that may exceed the expansion rate due to high recombination cross section. This process leads inevitably to annihilation and it may strongly suppress the abundance of these DM particles [35].

Since the Coulomb-like $y$-interaction is excluded for Majorana particles (in fact, ordinary Majorana mass term implies violation of $\mathrm{U}(1)$ gauge symmetry of $y$-interaction which is inconsistent with its long range nature (existence of massless dark photons)), it is assumed in our estimations that the considered DM particles are Dirac particles with mass $m \sim 100 \mathrm{GeV}$. If annihilating DM particles do not constitute all DM $\left(\Omega<\Omega_{\mathrm{CDM}}\right)$, then their contribution to density of clumps is assumed to be proportional to $\Omega / \Omega_{\mathrm{CDM}}$ (4). We do not specify annihilation channel of photon production, assuming that their averaged multiplicity for energy $E_{\gamma}>$ $100 \mathrm{MeV}$ is $N_{\gamma}=10$. It is quite typical value for high energy processes at respective energy release.

The photon flux at distance $l$ from the clump centre is given by

$$
F=\frac{P}{4 \pi l^{2}}=\frac{N_{\gamma}}{4 \pi l^{2}} \int_{V}\left\langle\sigma_{\mathrm{ann}} v\right\rangle n \bar{n} d V,
$$

where the particles/antiparticles number density is

$$
n=\bar{n}=\frac{1}{2} \frac{\rho(r)}{m} \times \frac{\Omega}{\Omega_{\mathrm{CDM}}} .
$$

Note that the fraction of subdominant DM particles should be suppressed in the clumps of mass $M<M_{\min }$, where $M_{\min }$ 
is the minimal mass which could be formed by considered DM particles if they prevailed in density. In our study we do not take into account this. The value $\left\langle\sigma_{\text {ann }} v\right\rangle$ is determined by averaging over velocity distribution of DM particles inside the clump, assumed to be Maxwellian one with the "virial" temperature $T_{\text {vir }}=G M m / 2 R$.

LAT registers $\gamma$-radiation with energy $E_{\gamma}>100 \mathrm{MeV}$ [56] and the flux $>F_{\min } \approx 3 \times 10^{-9} \mathrm{~cm}^{-2} \mathrm{sec}^{-1}$. The value $F_{\min }$ determines the maximal distance $l_{\max }$ at which the clump can be registered as $\gamma$-source. It gives for chosen profile $l_{\max } \sim$ $10^{-5} \mathrm{pc}$ for $\sigma_{0}=10^{-35} \mathrm{~cm}^{2}$ and $10^{-10} M_{\odot}$ without $y$-interaction and $l_{\max } \sim 1 \mathrm{pc}$ with $y$-interaction and the same parameters. The last magnitude corresponds to $\alpha \sim 10^{-2}$ and changes as $\propto \sqrt{\alpha}$ for $\alpha \ll 10^{-2}$ and $\propto 1 / \sqrt{\alpha}$ for $\alpha \gg 10^{-2}$. All the obtained $l_{\text {max }} \ll$ Galactic size, which justifies assumption that clump number density $n_{\mathrm{cl}} \approx$ const and corresponds to the local one. So

$$
n_{\mathrm{cl}}=\frac{\xi \rho_{\mathrm{loc}}}{M} \approx 1.6 \times 10^{-5} \frac{M_{\odot}}{M} \mathrm{pc}^{-3},
$$

where $\rho_{\text {loc }}=0.3 \mathrm{GeV} / \mathrm{cm}^{3}$. The number of clumps which may be detected by LAT is

$$
N_{\mathrm{cl}}=n_{\mathrm{cl}} \times \frac{4}{3} \pi l_{\max }^{3}
$$

Note that the given results turn out to be independent of $M$. It is not so for other laws of velocity dependence of cross section [31-33].

The analogous results for some other profile models are given in [33].

Since the clumps at distance $l<l_{\max }$ are expected to be distributed homogeneously, we can try to explain by them only isotropic component of unidentified PGS registered by Fermi LAT [57]. It includes $\sim 100$ sources. From this, respective regions of the parameters $\alpha$ and $\sigma_{0}$ for the typical clump masses $10^{-10} \div 10^{-6} M_{\odot}$ [22] are obtained. The results are shown at Figure 1. Note that unidentified PGS data also allow another application to the exotic physics [58, 59].

The distant clumps situated at $l>l_{\max }$ should contribute to the diffuse $\gamma$-radiation. $\gamma$-flux from them per given solid angle can be expressed as

$$
\Phi=\int_{l_{\max }}^{l_{\text {halo }}} F n_{\mathrm{cl}} l^{2} d l=\frac{P n_{\mathrm{cl}} l_{\text {halo }}^{\mathrm{eff}}}{4 \pi},
$$

where $F$ and $P$ are introduced in (3), $l_{\text {halo }}$ is the distance to the edge of halo along line of sight, and $l_{\text {halo }}^{\text {eff }} \approx 10 \mathrm{kpc}$ is its effective value (typical for many halo density profiles (for direction opposite Galactic center (where flux is more relevant to be compared with extracted by Fermi/LAT isotropic component of gamma background) this value varies from 6 to $11 \mathrm{kpc}$ depending on profile and is almost insensitive to $\left.\left.l_{\max }\right)\right) ; l_{\max }$ is negligible with respect to $l_{\text {halo }}$. One requires that

$$
\Phi<\Phi_{\exp } \approx 1.5 \times 10^{-5} \mathrm{~cm}^{-2} \mathrm{~s}^{-1} \mathrm{sr}^{-1}
$$

where $\Phi_{\text {exp }}$ is the diffuse $\gamma$-background measured by LAT [60]. It puts, as in the case of PGS, the lower constraint

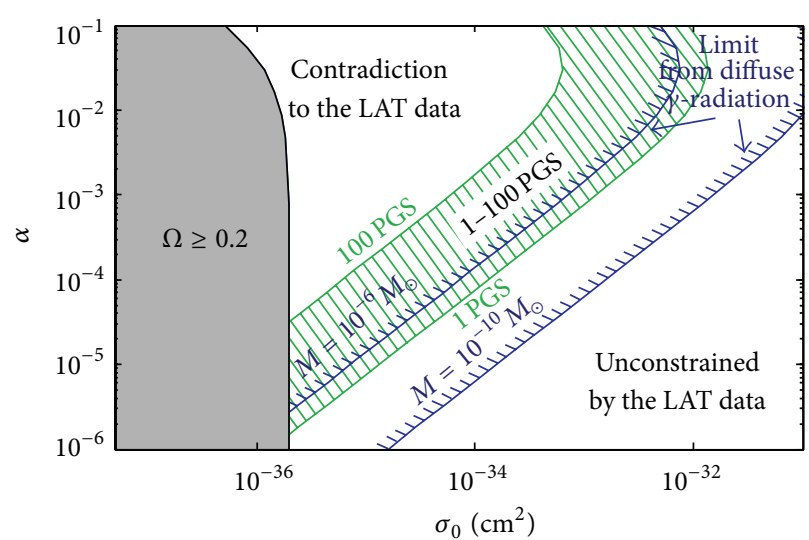

FIGURE 1: Allowed and forbidden regions of parameters $\alpha$ and $\sigma_{0}$ are shown as they are obtained on the base of Fermi LAT data on PGS and diffuse $\gamma$-radiation.

on cross section parameter $\sigma_{0}$ and upper constraint on parameter $\alpha$, which are also plotted on Figure 1. It follows from these constraints that the possibility to have PGS for clump mass $M=10^{-10} M_{\odot}$ is completely ruled out, while such possibility for $M=10^{-6} M_{\odot}$ is constrained but up to $\sim 10$ PGS is still possible. Higher masses of clumps avoid these restrictions.

As to the parameters $\sigma_{0}$ and $\alpha$, the ranges $3 \times 10^{-36} \lesssim$ $\sigma_{0} \lesssim 10^{-32} \mathrm{~cm}^{2}$ and $10^{-6} \leqslant \alpha \lesssim 10^{-1}$ are found to be the most interesting (Figure 1). Note that given range virtually excludes considerable contribution into $\sigma_{0}$ of the annihilation channel into $y$-photons. In fact if DM particles are $s=1 / 2$ Dirac particles, then this contribution is $\pi \alpha^{2} / m^{2}$ being much less than the obtained region of $\sigma_{0}$.

The obtained results can be generalized for a case of arbitrary values of the used parameters by multiplying $\sigma_{0}$ on Figure 1 by $\zeta$ :

$$
\begin{aligned}
\zeta= & \left(\frac{m}{100 \mathrm{GeV}}\right)^{2}\left(\frac{\Omega_{\mathrm{CDM}}}{0.2}\right)^{2}\left(\frac{10}{N_{\gamma}}\right) \\
& \times\left(\frac{F_{\min }}{3 \times 10^{-9} \mathrm{~cm}^{-2} \sec ^{-1}}\right)\left(\frac{0.002}{\xi}\right)^{2 / 3} .
\end{aligned}
$$

Effects of DM annihilation during period of the recombination of hydrogen can put constraints on the parameters of $y$-interaction. However, this question should be considered along with effects of DM recombination which might help to escape these constraints, which is out of the scope of the present paper.

It is interesting to note that one of the subdominant dark matter candidates, heavy neutrinos $v_{4}$ with $y$-interaction, can explain a part of unidentified point-like LAT sources due to $v_{4} \bar{v}_{4}$-annihilation with mass $m_{v_{4}} \sim 46-49 \mathrm{GeV}$ [3133]. At Figure 2 a typical $\gamma$-spectrum from $47 \mathrm{GeV}$ neutrinos annihilation is shown in comparison with measured spectrum of one of the nonidentified PGS (annihilation spectrum was obtained with the help of Monte-Carlo generator Pythia 


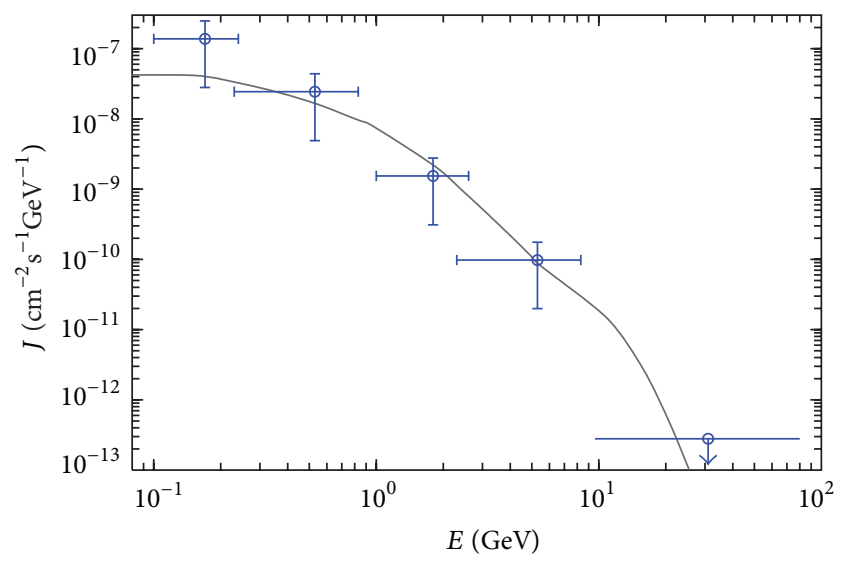

FIGURE 2: Expected spectrum from $v_{4} \bar{v}_{4}$-annihilation is shown for $m_{v_{4}}=47 \mathrm{GeV}$ in comparison with the observed spectrum of unidentified source 2FGL J1653.6-0159.

6.4 [61]). However, one should take into account that the heavy neutrino parameters are strongly restricted by underground experiments $[53,62]$, and the predicted $v_{4}$ relic density suffers with an uncertainty related with their possible annihilation due to recombination of the $y$-interacting neutrinos and antineutrinos after their freezing out in the early Universe [53].

\section{Conclusion}

In this paper we apply the approach developed earlier in our analysis of $\gamma$-radiation from annihilation of dark matter in clumps [31-33] to the case of new U(1) symmetry and put constraints on the parameters of the corresponding dark force from the data on discrete gamma sources and gamma background. We have shown that DM clumps in vicinity of the Solar System could be observed as pointlike sources of the $\gamma$-radiation and they can partially explain unidentified $\gamma$-sources, registered by LAT. Effects of DM annihilation in more distant clumps contribute to the diffuse gamma background and this contribution strongly depends on the minimal mass of the clumps. The smaller the mass of clumps the stronger the effect of Sommerfeld-GamowSakharov enhancement of the annihilation rate so that the observational data on $\gamma$-background and on the unidentified point-like $\gamma$-sources provide constraints on the strength of the Coulomb-like dark force.

These constraints are highly sensitive to the choice of a density profile inside the clump and are obtained for the most "conservative" BGZ [21] model. It should be also noted that the suppression of the subdominant fraction of DM particles in clumps of mass $M<M_{\text {min }}$ has not been taken into account and requires special study. In addition the extragalactic clumps should give contribution in diffuse radiation, strengthening the constraint somewhat [63], while observational limits on $\gamma$-flux from separate galaxies or their clusters may become evaded [64].

\section{Conflict of Interests}

The authors declare that there is no conflict of interests regarding the publication of this paper.

\section{Acknowledgments}

The authors express gratitude to V. Dokuchaev and Yu. Eroshenko for useful discussion of clump evolution. The work of A. Kirillov was supported by Grant of RFBR no. 14-0231417.

\section{References}

[1] B. W. Lee and S. Weinberg, "Cosmological lower bound on heavy-neutrino masses," Physical Review Letters, vol. 39, no. 4, pp. 165-168, 1977.

[2] D. A. Dicus, E. W. Kolb, and V. L. Teplitz, "Cosmological upper bound on heavy-neutrino lifetimes," Physical Review Letters, vol. 39, no. 4, pp. 168-171, 1977.

[3] D. A. Dicus, E. W. Kolb, and V. L. Teplitz, "Cosmological implications of massive, unstable neutrinos," The Astrophysical Journal, vol. 221, pp. 327-341, 1978.

[4] J. E. Gunn, B. W. Lee, I. Lerche, D. N. Schramm, and G. Steigman, "Some astrophysical consequences of the existence of a heavy stable neutral lepton," The Astrophysical Journal, vol. 223, pp. 1015-1031, 1978.

[5] F. W. Stecker, "The cosmic gamma-ray background from the annihilation of primordial stable neutral heavy leptons," The Astrophysical Journal, vol. 223, pp. 1032-1036, 1978.

[6] Ya. B. Zeldovich, A. A. Klypin, M. Yu. Khlopov, and V. M. Chechetkin, "Astrophysical restrictions of the heavy stable neutral leptons mass," Yadernaya Fizika, vol. 31, pp. 1286-1294, 1980.

[7] J. Silk and M. Srednicki, "Cosmic-ray antiprotons as a probe of a photino-dominated universe," Physical Review Letters, vol. 53, no. 6, pp. 624-627, 1984.

[8] R. V. Konoplich and M. Yu. Khlopov, "Astrophysical constraints on the mass of very heavy stable neutrinos," Physics of Atomic Nuclei, vol. 57, pp. 425-431, 1994.

[9] F. W. Stecker, S. Rudaz, and T. F. Walsh, "Galactic antiprotons from photinos," Physical Review Letters, vol. 55, no. 23, pp. 26222625, 1985.

[10] S. Rudaz and F. W. Stecker, "Cosmic-ray antiprotons, positrons, and gamma rays from halo dark matter annihilation," The Astrophysical Journal, vol. 325, pp. 16-25, 1988.

[11] J. E. Gunn, "Massive galactic halos. I-formation and evolution," The Astrophysical Journal, vol. 218, pp. 592-598, 1977.

[12] S. D. M. White and M. J. Rees, "Core condensation in heavy halos: a two-stage theory for Galaxy formation and clustering," Monthly Notices of the Royal Astronomical Society, vol. 183, pp. 341-358, 1978.

[13] P. J. E. Peebles, "Large-scale background temperature and mass uctuations due to scale-invariant primeval perturbations," The Astrophysical Journal, vol. 263, pp. L1-L5, 1982.

[14] G. R. Blumenthal, H. Pagels, and J. R. Primack, "Galaxy formation by dissipationless particles heavier than neutrinos," Nature, vol. 299, no. 5878, pp. 37-38, 1982.

[15] G. R. Blumenthal, S. M. Faber, J. R. Primack, and M. J. Rees, "Formation of galaxies and large-scale structure with cold dark matter," Nature, vol. 311, no. 5986, pp. 517-525, 1984. 
[16] A. V. Gurevich and K. P. Zybin, "Large-scale structure of the Universe. Analytic theory," Physics-Uspekhi, vol. 38, no. 7, pp. 687-722, 1995.

[17] J. Ellis, R. A. Flores, K. Freese, S. Ritz, D. Seckel, and J. Silk, "Cosmic ray constraints on the annihilations of relic particles in the galactic halo," Physics Letters B, vol. 214, no. 3, pp. 403412, 1988.

[18] M. Kamionkowski and M. S. Turner, "Distinctive positron feature from particle dark-matter annihilations in the galatic halo," Physical Review D, vol. 43, no. 6, pp. 1774-1780, 1991.

[19] V. S. Berezinsky, A. V. Gurevich, and K. P. Zybin, "Distribution of dark matter in the galaxy and the lower limits for the masses of supersymmetric particles," Physics Letters B, vol. 294, no. 2, pp. 221-228, 1992.

[20] A. V. Gurevich and K. P. Zybin, "The mass of cold dark matter particles and microlensing," Physics Letters A, vol. 208, no. 4-6, pp. 276-280, 1995.

[21] V. Berezinsky, V. Dokuchaev, and Y. Eroshenko, "Small-scale clumps in the galactic halo and dark matter annihilation," Physical Review D, vol. 68, no. 10, Article ID 103003, 19 pages, 2003.

[22] J. Diemand, B. Moore, and J. Stadel, "Earth-mass dark-matter haloes as the first structures in the early Universe," Nature, vol. 433, no. 7024, pp. 389-391, 2005.

[23] L. Pieri, E. Branchini, and S. Hofmann, "Difficulty of detecting minihalos via $\gamma$ rays from dark matter annihilation," Physical Review Letters, vol. 95, no. 21, Article ID 211301, 2005.

[24] J. Diemand, M. Kuhlen, and P. Madau, "Early supersymmetric cold dark matter substructure," Astrophysical Journal Letters, vol. 649, no. 1, pp. 1-13, 2006.

[25] L. Pieri, G. Bertone, and E. Branchini, "Dark matter annihilation in substructures revised," Monthly Notices of the Royal Astronomical Society, vol. 384, no. 4, pp. 1627-1637, 2008.

[26] A. V. Gurevich, K. P. Zybin, and V. A. Sirota, "Small-scale structure of dark matter and microlensing," Physics-Uspekhi, vol. 40, no. 9, pp. 869-898, 1997.

[27] S. M. Koushiappas, "Proper motion of gamma rays from microhalo sources," Physical Review Letters, vol. 97, no. 19, Article ID 191301, 2006.

[28] M. R. Buckley and D. Hooper, "Dark matter subhalos in the Fermi first source catalog," Physical Review D, vol. 82, no. 6, Article ID 063501, 2010.

[29] A. V. Belikov, M. R. Buckley, and D. Hooper, "Searching for dark matter subhalos in the Fermi-LAT second source catalog," Physical Review D, vol. 86, no. 4, Article ID 043504, 2012.

[30] H.-S. Zechlin and D. Horns, "Unidentified sources in the FermiLAT second source catalog: the case for DM subhalos," Journal of Cosmology and Astroparticle Physics, vol. 11, p. 50, 2012.

[31] K. M. Belotsky, A. A. Kirillov, and M. Yu. Khlopov, "Astrophysical manifestations of clumps of cold dark matter," Physics of Atomic Nuclei, vol. 76, no. 4, pp. 469-475, 2013.

[32] K. M. Belotsky, A. A. Kirillov, and M. Y. Khlopov, "Astrophysical manifestations of clumps of cold dark matter," Yadernaya Fizika, vol. 76, pp. 506-512, 2013.

[33] K. M. Belotsky, A. A. Kirillov, and M. Y. Khlopov, "Gammaray evidences of the dark matter clumps," Gravitation and Cosmology, vol. 20, pp. 47-54, 2014.

[34] K. M. Belotsky, M. Yu. Khlopov, and K. I. Shibaev, "Sakharov's enhancement in the effect of 4th generation neutrino," Gravitation and Cosmology, vol. 6, supplement 6, pp. 140-143, 2000.
[35] M. B. Konstantin, M. Yu. Khlopov, S. V. Legonkov, and K. I. Shibaev, "Effects of a new long-range interaction: recombination of relic heavy neutrinos and antineutrinos," Gravitation and Cosmology, vol. 11, pp. 27-33, 2005.

[36] K. N. Abazajian and J. P. Harding, "Constraints on WIMP and Sommerfeld-enhanced dark matter annihilation from HESS observations of the galactic center," Journal of Cosmology and Astroparticle Physics, vol. 2012, no. 1, article 041, 2012.

[37] T. R. Slatyer, N. Toro, and N. Weiner, "Sommerfeld-enhanced annihilation in dark matter substructure: consequences for constraints on cosmic-ray excesses," Physical Review D, vol. 86, no. 8, Article ID 083534, 18 pages, 2012.

[38] L. G. van den Aarssen, T. Bringmann, and Y. C. Goedecke, "Thermal decoupling and the smallest subhalo mass in dark matter models with Sommerfeld-enhanced annihilation rates," Physical Review D, vol. 85, no. 12, Article ID 123512, 20 pages, 2012.

[39] C. Armendariz-Picon and J. T. Neelakanta, "Structure formation constraints on Sommerfeld-enhanced dark matter annihilation," Journal of Cosmology and Astroparticle Physics, vol. 2012, no. 12, article 9, 2012.

[40] J. Chen and Y.-F. Zhou, "The $130 \mathrm{GeV}$ gamma-ray line and Sommerfeld enhancements," Journal of Cosmology and Astroparticle Physics, vol. 2013, no. 4, article 17, 2013.

[41] K. M. Belotsky, M. Yu. Khlopov, and K. I. Shibaev, "Composite dark matter and its charged constituents," Gravitation and Cosmology, vol. 12, pp. 93-99, 2006.

[42] D. E. Kaplan, G. Z. Krnjaic, K. R. Rehermann, and C. M. Wells, "Atomic dark matter," Journal of Cosmology and Astroparticle Physics, vol. 2010, no. 5, article 21, 2010.

[43] F.-Y. Cyr-Racine and K. Sigurdson, "Cosmology of atomic dark matter," Physical Review D, vol. 87, no. 10, Article ID 103515, 39 pages, 2013.

[44] T. Ishiyama, J. Makino, and T. Ebisuzaki, "Gamma-ray signal from Earth-mass dark matter microhalos," Astrophysical Journal Letters, vol. 723, no. 2, pp. L195-L200, 2010.

[45] M. Ackermann, A. Albert, L. Baldini et al., "Search for dark matter satellites using Fermi-LAT," The Astrophysical Journal, vol. 747, no. 2, article 121, 2012.

[46] G. Gamow, “Zur Quantentheorie des Atomkernes," Zeitschrift für Physik, vol. 51, no. 3-4, pp. 204-212, 1928.

[47] A. Sommerfeld, "Über die beugung und bremsung der elektronen," Annalen der Physik, vol. 403, no. 3, pp. 257-330, 1931.

[48] A. D. Sakharov, "Interaction of the electron and positron in pair production," Zhurnal Ėksperimental'noi i Teoreticheskoi Fiziki, vol. 18, pp. 631-635, 1948.

[49] J. B. Dent, S. Dutta, and R. J. Scherrer, "Thermal relic abundances of particles with velocity-dependent interactions," Physics Letters B, vol. 687, no. 4-5, pp. 275-279, 2010.

[50] J. Zavala, M. Vogelsberger, and S. D. M. White, "Relic density and CMB constraints on dark matter annihilation with Sommerfeld enhancement," Physical Review D, vol. 81, no. 8, Article ID 083502, 2010.

[51] J. L. Feng, M. Kaplinghat, and H.-B. Yu, "Sommerfeld enhancements for thermal relic dark matter," Physical Review D, vol. 82, no. 8, Article ID 083525, 2010.

[52] J. Zavala, M. Vogelsberger, T. R. Slatyer, A. Loeb, and V. Springel, "Cosmic X-ray and gamma-ray background from dark matter annihilation," Physical Review D, vol. 83, no. 12, Article ID 123513, 19 pages, 2011. 
[53] K. M. Belotsky, D. Fargion, M. Y. Khlopov, and R. V. Konoplich, "May heavy neutrinos solve underground and cosmic-ray puzzles?” Physics of Atomic Nuclei, vol. 71, no. 1, pp. 147-161, 2008.

[54] A. D. Dolgov and Y. B. Zeldovich, "Cosmology and elementary particles," Reviews of Modern Physics, vol. 53, no. 1, pp. 1-41, 1981.

[55] R. J. Scherrer and M. S. Turner, "On the relic, cosmic abundance of stable, weakly interacting massive particles," Physical Review D, vol. 33, no. 6, pp. 1585-1589, 1986.

[56] W. B. Atwood, A. A. Abdo, M. Ackermann et al., "The large area telescope on the Fermi Gamma-Ray Space Telescope mission," The Astrophysical Journal, vol. 697, no. 2, pp. 1071-1102, 2009.

[57] P. L. Nolan, A. A. Abdo, M. Ackermann et al., "Fermi large area telescope second source catalog," The Astrophysical Journal Supplement Series, vol. 199, no. 2, article 31, 2012.

[58] K. M. Belotsky, A. V. Berkov, A. A. Kirillov, and S. G. Rubin, "Black hole clusters in our Galaxy," Gravitation and Cosmology, vol. 17, no. 1, pp. 27-30, 2011.

[59] K. M. Belotsky, A. V. Berkov, A. A. Kirillov, and S. G. Rubin, "Clusters of black holes as point-like gamma-ray sources," Astroparticle Physics, vol. 35, no. 1, pp. 28-32, 2011.

[60] A. A. Abdo, M. Ackermann, M. Ajello et al., "Fermi large area telescope measurements of the diffuse gamma-ray emission at intermediate galactic latitudes," Physical Review Letters, vol. 103, no. 25, Article ID 251101, 6 pages, 2009.

[61] http://home.thep.lu.se/ torbjorn/Pythia.html.

[62] Z. Ahmed, D. S. Akerib, S. Arrenberg et al., "Search for weakly interacting massive particles with the first five-tower data from the cryogenic dark matter search at the soudan underground laboratory," Physical Review Letters, vol. 102, no. 1, Article ID 011301, 5 pages, 2009.

[63] K. C. Y. Ng, R. Laha, S. Campbell et al., "Resolving small-scale dark matter structures using multi-source indirect detection," http://arxiv.org/abs/1310.1915.

[64] M. A. Sanchez-Conde and F. Prada, "The attening of the concentration-mass relation towards low halo masses and its implications for the annihilation signal boost," http://arxiv.org/abs/1312.1729. 

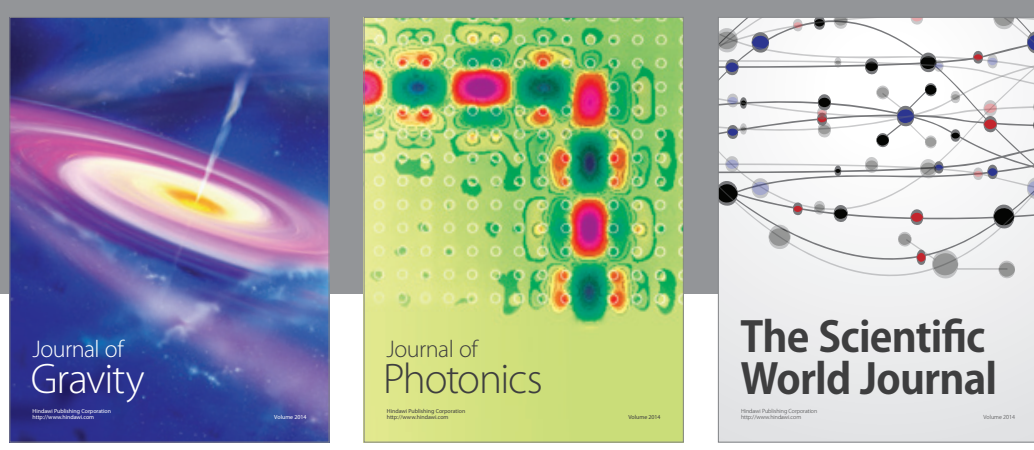

The Scientific World Journal
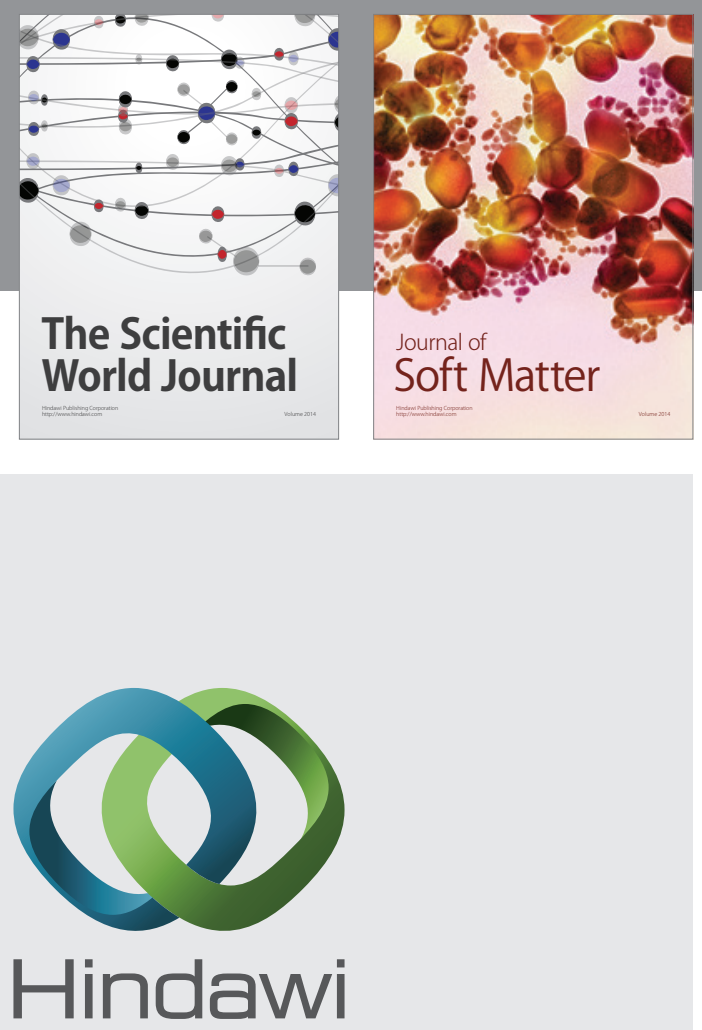

Submit your manuscripts at

http://www.hindawi.com

nternational Journal of

Statistical Mechanics
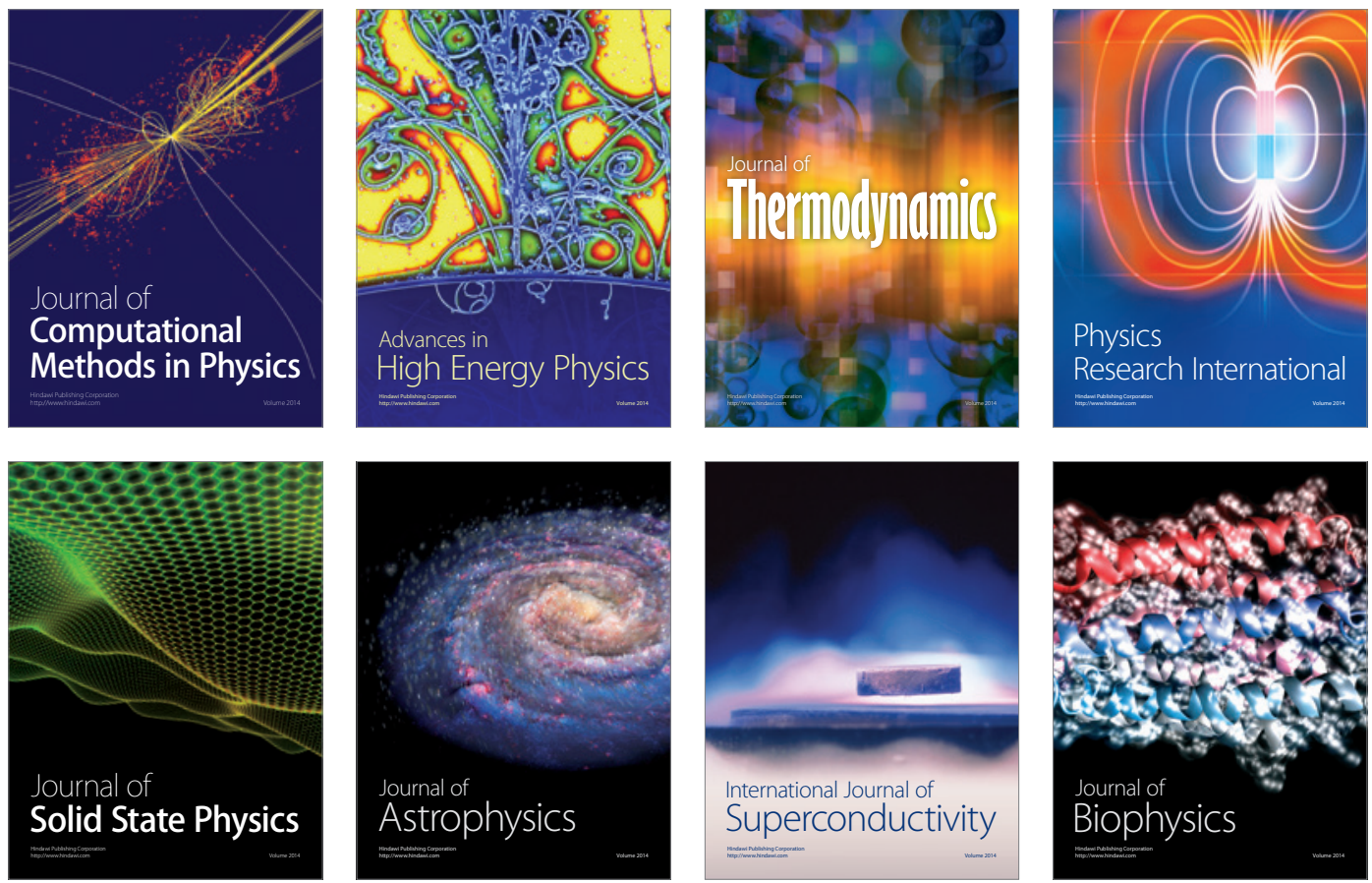
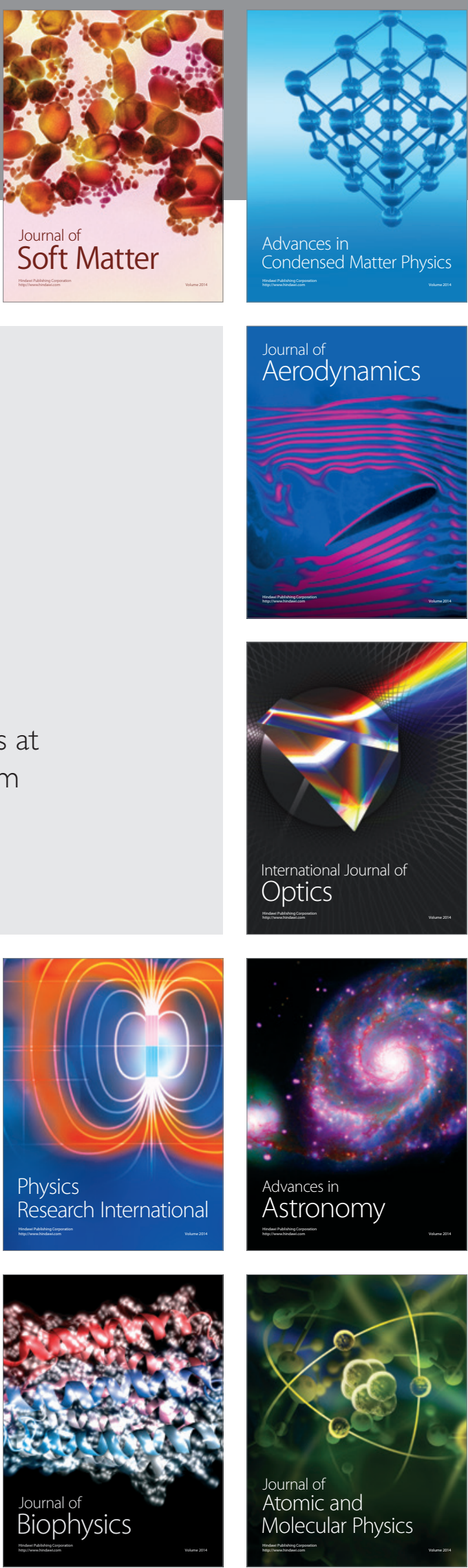